\title{
Design of IPv4/IPv6 Transition Strategy Based on NDN
}

\author{
Meigen Huang ${ }^{1, a}$, Yongzai $\mathrm{Si}^{2, b}$ \\ ${ }^{1}$ College of Computer Science and Technology, Chongqing University of Posts and \\ Telecommunications, Chongqing 400065, China; \\ ${ }^{2}$ College of Computer Science and Technology, Chongqing University of Posts and \\ Telecommunications, Chongqing 400065, China. \\ ahuangmg@cqupt.edu.cn, b499876464@qq.com
}

Keywords: IPv6 transition, NDN, IPv4, IPv6.

\begin{abstract}
The depletion of IPv4 addresses provides an opportunity to integrate IPv6 addresses into the network. But the IPv6 transition is an ongoing process rather than overnight. NDN is a new Internet architecture, based on Content-Centric Networking, data-oriented network or information-centric network, and solve the traditional IP and other contemporary Internet architecture unresolved issues. NDN uses hierarchically structured names, each packet adopts Type-Length-Value encoding. In this paper, we proposed IPv4/IPv6 transition strategy based on NDN, this method simplifies IPv6 transition and ensure safe transmission of data.
\end{abstract}

\section{Introduction}

The development of the Internet industry makes the IPv4 address quickly depleted, IPv6 as the next generation of Internet key technology [1], IPv6 has a larger number of addresses and higher security and other advantages. The two kinds of address protocols are not compatible with each other. Because most of the current backbone networks adopt IPv4, the smooth, efficient and stable transition of IPv4 to IPv6 is inevitable [2].

The dual stack technology provides a complete IPv4 and IPv6 protocol stack in the same network node, which facilitates communication between nodes using either protocol[3]. This is the most direct and effective way to transition from IPv4 to IPv6 because it avoids tunnel complexity, security, network latency, administrative overhead, and reduced path MTU. However, this is not always possible because many network devices may not support the IPv6 protocol stack.

At present, many Internet users do not support IPv4 / IPv6 dual protocol stack, so IPv4 nodes cannot directly access the IPv6 node. Therefore, you must use an IPv4 network infrastructure to host IPv6 packets. At this point, tunneling technology[4] is needed to realize the mutual exchange between different networks. Tunneling technology encapsulates IPv6 packets in IPv4, and actually uses IPv4 as the link layer of IPv6.

Network Address Translation[5] is a method of mapping an IP address space to another IP address space by modifying the network address information in the packet header of the Internet Protocol datagram, while passing the routing device. Protocol translation technology involves network layer, transport layer and application layer, including address translation, port conversion, TCP / UDP conversion, ICMP conversion and application layer translation. However, due to IPv4 and IPv6 protocol is not compatible, IPv4 packet fragmentation can be completed by the router and send host, and IPv6 packet fragmentation can only send the host to complete, but also to deal with the MTU and ICMP translation.

\section{Related work}

Yong Tang et al.[6] proposed IPv4 and IPv6 Network Interconnection Method Based on SDN. The control layer is designed for address translation module, translation module and other modules and needs to be combined with the DNS module. The control layer is responsible for address translation and protocol translation. The data layer is only responsible for forwarding data. Jing Huang et al.[7] 
proposed a general tunneling method based on SDN. The SDN controller accepts packets submitted by the switch, adds or removes tunnel information to the switch, and the switch performs the action according to the rules issued by the control layer. The method supports multiple tunneling techniques. Zhilan Huang et al.[8] proposed IPv4 and IPv6 conversion based on OpenFlow, an IPv6 user wants to access the IPv4 application, the query DNS obtains the corresponding IPv6 address, and reaches the OpenFlow network through the IPv6 network gateway. The controller sends the corresponding flow table to the OpenFlow switch, which forwards the packet to the server. The server invokes the protocol conversion module and returns the data to the IPv6 user.

The introduction of SDN adds a new method in the address protocol transition. OpenFlow[9] 1.0 provide three options such as port-based, layering and hybrid. OpenFlow version above 1.2 version also supports IPv4 and IPv6 protocols to ensure a smooth transition from IPv4 to IPv6 .However, in the process of IPv4 to IPv6 transition, for example, in the tunneling technology, the packet is encapsulated at the network boundary, and the packet is also debuted at the network boundary. Also in the network address translation, the address translator for the data packet translation, protocol translation and other operations, the packet processing is still at the network boundary. However, SDN-based IPv4 / IPv6 transition strategy to the packet address translation, protocol translation and other operations to the controller processing, the two-tier exchange is only responsible for packet forwarding, these transition strategies not only complicated the process of address transition, and not easy to accomplish.

\section{Design of IPv4 / IPv6 Transition Strategy Based on NDN}

NDN and Address transition. Named Data Networking (NDN)[10] is a new Internet architecture, based on Content-Centric Networking (CCN), data-oriented network or information-centric network, and solve the traditional IP and other contemporary Internet architecture unresolved issues.

NDN and address transition have the following advantages. First of all, NDN uses hierarchically structured names, e.g. , a video may have the name/sin/videos/WidgetA.mpg, compared with the traditional IP network, there is no problem of address exhaustion. Furthermore, every packet in NDN adopts Type-Length-Value (TLV) encoding, NDN packet format does not have a fixed packet header, nor does it encode the protocol version number, which can solve different MTU problems of different network. At length, in the NDN, by signing and verifying any named data, ensure data transmission at the network layer.

Design of the transition strategy. DNS has well established name space allocation procedures and governance bodies. NDN can use this existing application name space for data delivery. The transition strategy is shown in Figure 1. IPv4 network side and IPv6 network side have DNS server, both sides of the DNS can communicate through the NDN network. DNS server can record the domain name, IPv4, IPv6 mapping, and the query to the IPv4 address into an IPv6 address, the same can query to the IPv6 address mapping to a public IPv4 address.

The border device of the NDN network must be able to identify specific packets, such as IPv6 addresses with specific prefixes or a specific IPv4 public network address. When packets with IPv6 network side need to access IPv4 through NDN, DNS will query the IPv4 address into a specific prefix with the IPv6 address, the prefix can be NDN network boundary device identification. Also, when a packet on the IPv4 network side needs to pass through the NDN and access IPv6, the DNS will return a specific public IPv4 address, which can be identified by the NDN network boundary.

NDN network boundary device to identify the packet, encapsulate the entire packet, and record the IPv6 source address--domian.X--IPv4 source address and IPv6 source address--domain.y--IPv6 destination address. When the packet arrives through the NDN to the network boundary device, it searches the domain name and sets new IP address for the packet according to the mapping relationship between the domain--IPv4 address--IPv6 address. 


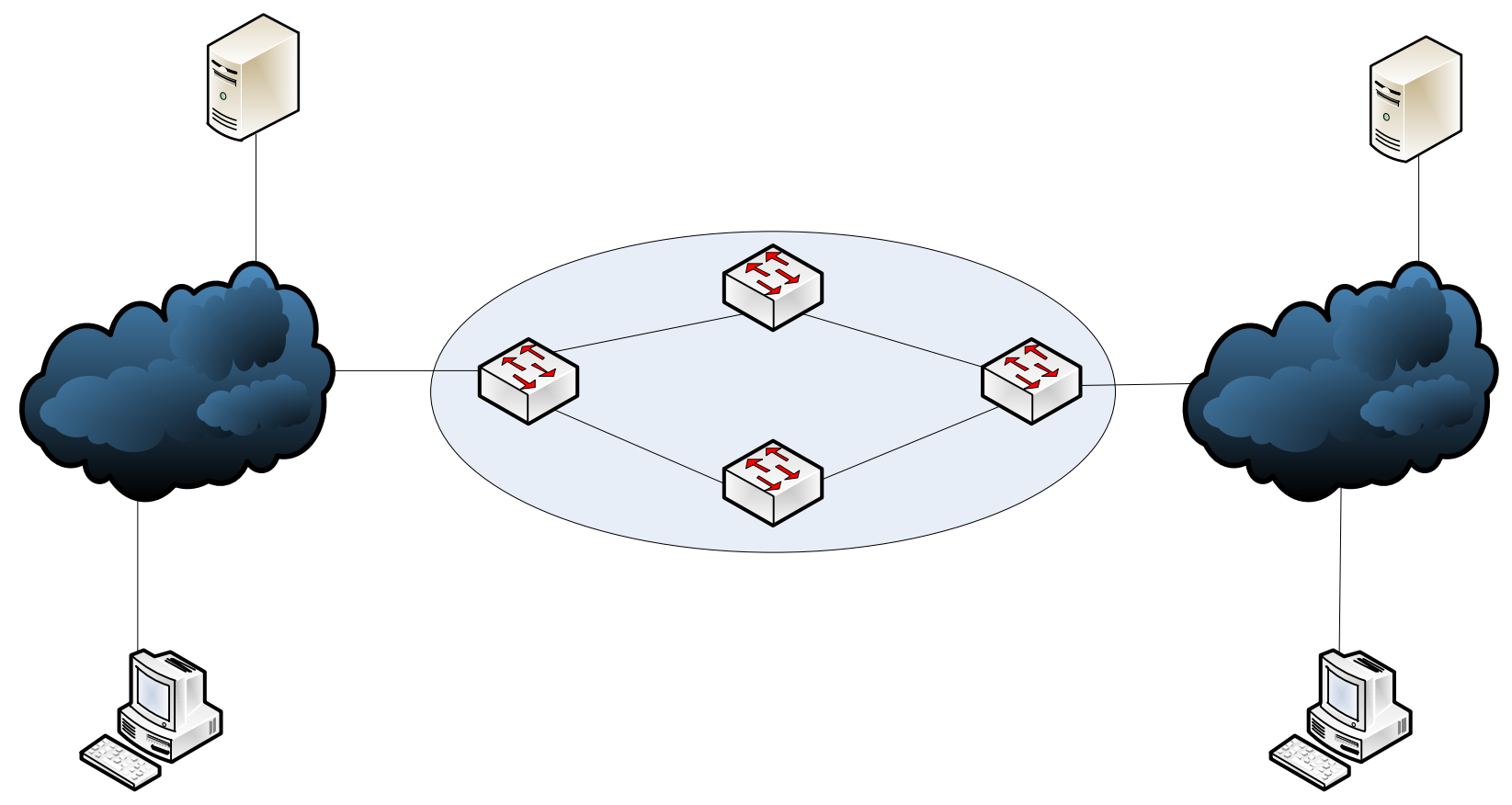

Fig. 1 IPv4/IPv6 transition strategy based on NDN

IPv6 node establishes communication with IPv4 network. The steps by which Host1 establishes communication with Host2 are :

1.Host1 initiates DNS request. If the resolution is successful, return the resolved IPv6 address to Host1;

2. If the source DNS resolves to fail, send the domain name DNS request to the IPv4 network side through the NDN;

3. If the IPv4 side DNS resolution is successful, will be resolved after the A record sent to the IPv6 side of the DNS; if the query fails, then return NULL;

4. IPv6 side DNS will record A synthesis AAAA records, that is, IPv4 addresses into IPv6 addresses (IPv4 address to add a specific prefix), and the address back to Host1;

5.Host1 initiates communication with the synthesized IPv6 address as the destination address. The packet arrives at the NDN network boundary. The border router identifies the special address prefix and records the mapping relationship, that is, the domain name-IPv6-IPv4 mapping, encapsulates the IPv6 packet into NDN packet format, packets through the NDN network to reach the border router;

6. The NDN border router searches the domain name-IPv6-IPv4 mapping table, decapsulates the NDN packet and sets the corresponding IPv4 destination address and source address according to the mapping table.

7. The IPv4 packet arrives at Host2 through the IPv4 network.

IPv4 node establishes communication with IPv6 network. The steps by which Host2 establishes communication with Host2 are :

1.Host2 initiates DNS request, if the resolution is successful, return to the resolved IPv4 address to Host2;

2. If the DNS resolution fails, send the domain name DNS request to the IPv6 network side DNS through NDN;

3. If the IPv6 side DNS resolves successfully, the parsed AAAA record is sent to the IPv4 side DNS; if the query fails, it returns NULL;

4. IPv4 side DNS will return the AAAA records are mapped to IPv4 address, this IPv4 address to return to Host2, and record this mapping;

5.Host1 initiates communication with the received IPv4 address as the destination address. The packet reaches the NDN network boundary. The border router identifies the destination address and records the mapping relationship, that is, the domain name-IPv6-IPv4 mapping table, encapsulates 
the IPv4 packet into NDN data Packet format, packets through the NDN network to reach the border router;

6. The border router searches the domain name-IPv6-IPv4 mapping table, decapsulates the NDN packet, and sets the corresponding IPv6 destination address and source address according to the mapping table.

7. The IPv6 packet arrives at Host1 through the IPv6 network.

\section{Summary}

IPv6 has quietly integrated into the information age of the Internet.But, IPv4 and IPv6 is not compatible, there is no flay day and transition is really difficult. What we have to do is try to reduce the complexity of IPv6 transition, to ensure efficient and stable transition. We proposed IPv4/IPv6 transition strategy based on NDN, this method not only can be safe and efficient transmission of data packets, but also introduce NDN into the Internet.

\section{References}

[1] W.Stallings.IPv6: the new Internet protocol[J].IEEE Journals \& Magazines, 1996,34(7):96-108.

[2] J.Gnana Jayanthi, S.Albert Rabara.IPv4 addressing architecture in IPv6 network[C].IEEE Conference Publications, 2010, 282-287.

[3] E. Nordmark, R. Gilligan. Basic Transition Mechanisms for IPv6 Hosts and Routers[S].RFC 4213,2005.

[4] S. Krishnan, D. Thaler, J. Hoagland. Security Concerns with IP Tunneling[S].RFC 6169,2011.

[5] P.Srisuresh, K. Egevang. Traditional IP Network Address Translator (Traditional NAT)[S].RFC 3022, 2001.

[6] Y.Tang, WenYong Wang. A Method of IPv4 and IPv6 Interconnection Based on SDN.CN Patennt,104734963 A.(2015).

[7] J.Huang,T. Zhou. A general tunneling method based on SDN,CN Patent,105409167.(2016).

[8] Zhilan Huang,Nan Chen. IPv4 and IPv6 conversion based on OpenFlow. China Academic Journal Electronic Publishing House,Z2.104,2014.

[9] Information on http://archive.openflow.org/

[10] L. Zhang et al. Named data networking (NDN) project[R]. Technical Report NDN-0001,2010. 